\title{
On Distributed Localization for Road Sensor Networks: A Game Theoretic Approach
}

\author{
Jie Jia, Guiyuan Zhang, Xingwei Wang, and Jian Chen \\ College of Information Science and Engineering, Northeastern University, Shenyang 110819, China \\ Correspondence should be addressed to Xingwei Wang; wangxw@mail.neu.edu.cn
}

Received 7 October 2013; Accepted 1 December 2013

Academic Editor: Xinjie Zhang

Copyright (C) 2013 Jie Jia et al. This is an open access article distributed under the Creative Commons Attribution License, which permits unrestricted use, distribution, and reproduction in any medium, provided the original work is properly cited.

\begin{abstract}
Road sensor network is an important part of vehicle networks system and is critical for many intelligent automobile scenarios, such as vehicle safety monitoring and transportation efficiency supporting. Localization of sensors is an active and crucial issue to most applications of road sensor network. Generally, given some anchor nodes' positions and certain pairwise distance measurements, estimating the positions of all nonanchor nodes embodies a nonconvex optimization problem. However, due to the small number of anchor nodes and low sensor node connectivity degree in road sensor networks, the existing localization solutions are ineffective. In order to tackle this problem, a novel distributed localization method based on game theory for road sensor networks is proposed in this paper. Formally, we demonstrate that our proposed localization game is a potential game. Furthermore, we present several techniques to accelerate the convergence to the optimal solution. Simulation results demonstrate the effectiveness of our proposed algorithm.
\end{abstract}

\section{Introduction}

A wireless sensor network (WSN) usually consists of a large number of static sensor nodes that organize themselves into multihop networks [1]. Sensor nodes are able to measure various parameters of the environment and transmit collected data to the sink node through multihop communication. Once the sink node received the sensed data, it processes and forwards it to the users. Roadways, which are mainly used for the transportation of people, are one of the typical application areas for WSNs. Recently, roadways equipped with intelligent sensor nodes have prompted the emergence of road sensor network, which is a new type of WSN and is considered as a promising solution to effectively enhance the driving safety and mobility [2]. Furthermore, the U.S. Department of Transportation (DOT) and many automobile companies (e.g., General Motors and Toyota) have started to apply the WSN technology to the Intelligent Transportation Systems (ITS) infrastructures [3-5]. In addition, the European Telecommunications Standards Institute (ETSI) has been making globally applicable standards for Intelligent Transportation Systems integrated with WSNs [6], in which sensor nodes are deployed on the roadways to monitor the road condition (e.g., road construction sites or obstacles) and to announce such condition to vehicles through vehicle-tovehicle or infrastructure-to-vehicle communications.

Localization technology has become one of the key technologies of road sensor networks [7]. Firstly, node localization is crucial for potential location-aware applications of ITS, such as surveillance and target tracking. Secondly, node location is needed for geographic message forwarding, geographic hash tables, coverage control, energy conservation algorithms, and others [7-9]. Finally, security can also be enhanced by location awareness [10]. In wireless sensor networks, many localization approaches have been proposed. These algorithms can be classified into coarseand fine-grained localization, respectively. The former relies on lower hardware but is less accurate, while the latter facilitates higher precision of position determination and may represent the most suitable ones. In these fine-grained localization techniques, only a fraction of the nodes, named as anchor nodes, are endowed with their exact coordinates through GPS or manual placement, while the remaining nodes, referred to as nonanchor nodes, are able to calculate their distances to nearby nodes through measurement-based techniques, such as received signal strength (RSS) [11], angle 
of arrival (AoA) [12], or time of arrival (ToA) [13]. Hence, assuming that the coordinates of anchor nodes are known and obtaining pairwise distance measurements between nearby nodes, the fine-grained localization problem is to estimate the positions of all nonanchor nodes. It is important to recognize that this problem can be formulated as multivariable nonconvex optimization problem [14], which has proven to be rather difficult [15]. In response to a multivariable nonconvex optimization task, three different approaches have been presented, namely, multidimensional scaling (MDS) [16], semidefined programming (SDP) [17], and stochastic optimization $[14,18]$. MDS, applied widely in data analysis, hinges on obtaining the relative coordinates of each node based on a starting distance matrix. On the other hand, SDP relaxes the original nonconvex optimization problem and obtains an approximate solution at a reduced computational effort. Since the relaxation may increase estimation error, additional refinements, such as gradient descent search procedures, are often employed to refine the initial solutions obtained by SDP [19]. Finally, the third class of techniques considers the heuristic optimization algorithms to efficiently solve the localization problem, such as simulated annealing (SA), genetic algorithms, or pareto archived evolution strategies (PAES). However, all these methods discussed above cannot be directly applied to road sensor networks, which is mainly because that these methods ignore two important characteristics in road networks. One is that; in road sensor networks, sensors are deployed with only a small number of anchor nodes; the other is that the localization estimation in road sensor networks should be executed in a distributed manner, and previous works using centralized localization may result in severe communication congestion and high computational complexity. Although some distributed finegrained localization methods (e.g., distributed SDP [20] and distributed MDS [21]) have been proposed to improve network performance recently, however, existing distributed methods tend to have worse localization accuracy than centralized methods, which are further exacerbated by the fact that the number of anchor nodes is sufficiently small and the network connectivity degree is not sufficiently high. Hence, exploiting distributed localization algorithm with high localization accuracy guarantee is of great importance for road sensor networks.

In this work, we develop a computationally efficient and distributed localization method for road sensor network. The proposed method borrows the model from game theory, which is a mathematical tool, particularly useful in the network engineering field to model highly complex scenarios. This mathematical tool provides researchers with the ability to model individual or independent decision makers called "players." Every player interacts with other players and has an impact on their decisions. The dynamics of the localization, in which pure mathematical analysis has been met with limited success, closely resemble this observation. It has recently come to our attention that some works have also applied game theory for WSNs [22, 23]. In [22], the authors proposed a localization game in wireless sensor networks, where the two-player game model is used to assess the reliability of the node localization data, thus identifying the attacker node and enhancing the security of WSNs. In [23], the coalition game theory is applied to form coalitions, which can reduce communication costs and track mobile target. The major differences between their work and ours lie, however, in that, (i) in our work, we intend to achieve a fully distributed localization with high localization accuracy. We have given a theoretical proof on the fact that the localization game is a potential game; (ii) our distributed sensor localization algorithm is based on the above theoretical analysis and is hence theoretically founded; (iii) we have presented new methods to accelerate the convergence for the localization game.

The rest of the paper is organized as follows. Section 2 presents the assumptions, network model, and design considerations. A fully distributed localization scheme based on potential game is proposed in Section 3. Section 4 gives the simulation results, and Section 5 concludes our paper.

\section{Network Model}

In this section, we present our network model and basic assumptions. Consider a 2D road sensor network composed of $N$ sensor nodes with position $p_{i}=\left(x_{i}, y_{i}\right) \in R^{2}, i=$ $1, \ldots, N$, where 1 to $N_{n}\left(N_{n}<N\right)$ are nonanchor nodes to be localized and $N_{n+1}$ to $N$ are anchor nodes whose positions are known a priori. We adopt a simple disk model for network connectivity determination, and if the distance between nodes $i$ and $j$, denoted by $r_{i j}$, is less than the physical communication range $R_{c}$, we say that nodes $i$ and $j$ are neighbor nodes, and thus can communicate and obtain a (noisy) distance measurement $d_{i j}$ using some measurement technique (see Section 1), and it is represented as

$$
d_{i j}=r_{i j}+e_{i j}
$$

where $r_{i j}=\left\|p_{i}-p_{j}\right\|$ represents the true distance between $i$ and $j$ (\| $\|$ denotes the norm-2 operator) and $e_{i j}=e_{j i}$ is the corresponding measurement error. The statistics on this measurement error depend on the ranging technique used in [24]. Considering that the RSS-based distance estimation can easily be achieved in most modern wireless systems, the measurement error model used in our paper is similar to $[15,18,19]$, which are independent and follow a zeromean Gaussian distribution with an unknown variance $\sigma^{2}$, $e_{i j} \sim N\left(0, \sigma_{i j}^{2}\right)$. Further, we assume range measurements are symmetric; that is, $d_{i j}=d_{j i}$, for all $i, j$.

Based on the above disk model, each node can easily determine which other nodes it can directly communicate with, and we define $N_{i}$ as the neighbor set of node $i$, and it can be represented as

$$
N_{i}=\left\{j \in N, j \neq i:\left\|p_{i}-p_{j}\right\| \leq R_{c}\right\} .
$$

Without loss of generality, we assume the position of nonanchor node $i \in\left\{1, \ldots, N_{n}\right\}$ that is estimated by the localization algorithm as $\widehat{p}_{i}=\left\{\widehat{x}_{i}, \widehat{y}_{i}\right\}$, so that the estimated 
distance $d\left(\widehat{p}_{i}, \widehat{p}_{j}\right)$ between two neighbor sensors $i$ and $j$ can be calculated as

$$
d\left(\widehat{p}_{i}, \widehat{p}_{j}\right)= \begin{cases}\left\|\hat{p}_{i}-p_{j}\right\|, & \text { if } j \text { is an anchor node }, \\ \left\|\widehat{p}_{i}-\widehat{p}_{j}\right\|, & \text { otherwise. }\end{cases}
$$

The difference of $d_{i j}$ and $d\left(\widehat{p}_{i}, \widehat{p}_{j}\right)$ is denoted by $\delta\left(\widehat{p}_{i}, \widehat{p}_{j}\right)$ and is determined as follows:

$$
\begin{aligned}
\delta & \left(\widehat{p}_{i}, \widehat{p}_{j}\right) \\
& = \begin{cases}\left(d_{i j}-d\left(\widehat{p}_{i}, \widehat{p}_{j}\right)\right)^{2}, & \text { if nodes } i \text { and } j \text { are neighbors, } \\
0, & \text { otherwise. }\end{cases}
\end{aligned}
$$

If all the nonanchor nodes are localized accurately, $\delta\left(\widehat{p}_{i}, \widehat{p}_{j}\right)$ tends to 0 . Obviously, the goal of the localization algorithm is to estimate the position of all nonanchor nodes as accurately as possible, and it can be defined as the following optimization problem:

$$
\operatorname{Min} U_{\mathrm{Net}}=\sum_{i=1}^{N_{n}} \sum_{j \in N_{i}} \delta\left(\widehat{p}_{i}, \widehat{p}_{j}\right) .
$$

To this purpose, for any nonanchor node $i$, its own utility function can be defined as

$$
\operatorname{Min} \sum_{j \in N_{i}} \delta\left(\widehat{p}_{i}, \widehat{p}_{j}\right)
$$

\section{Distributed Localization Based on Game Theory}

In this section, we firstly present the distributed localization scheme applying the game theory and then present the techniques that can be adopted to improve convergence speed. The main objective of such modeling is to achieve the precise localization using the mathematical analyses provided by the game theory framework.

In our algorithm, each nonanchor node is considered as a decision maker in the game. All the game players, denoted by $A=\left\{1,2, \ldots, N_{n}\right\}$, have a common strategy space $\mathbf{S}=\left\{s_{i}\right\}$, for all $i \in A$. In this context, we map the estimated position as its chosen strategy, and the strategy of the player $i$ is its estimated location $s_{i}=\left\{\widehat{x}_{i}, \widehat{y}_{i}\right\}$. The game profile is defined as the Cartesian product of the players' strategy vector, $\Psi=$ $\times_{i \in A} s_{i}=s_{1} \times s_{2} \times \cdots \times s_{N_{n}}$. Note that a game profile includes one strategy for each player. Similarly, $s_{-i}$ is defined as the strategy set chosen by all the other players except player $i$.

We assume that each player has a decision module to select its location based on its local information and observation. More specifically, we consider a practical scenario where each player only knows the information of its neighbors. Therefore, for each player, say $i$, the location $s_{i}=\left\{\widehat{x}_{i}, \widehat{y}_{i}\right\}$ should be selected to minimize the following:

$$
U_{i}(\Psi)=U_{i}\left(s_{i}, s_{-i}\right)=\sum_{j \in N_{i}} \delta\left(\widehat{p}_{i}, \widehat{p}_{j}\right),
$$

where $U_{i}(\Psi)$ is defined as the utility function of player $i \in A$.
From the network's perspective, it is desirable to obtain the optimum location selection $\Psi^{*}$ which minimizes the network cost function given by

$$
R(\Psi)=\sum_{i=1}^{N_{n}} \sum_{j \in N_{i}} \delta\left(\widehat{p}_{i}, \widehat{p}_{j}\right)
$$

In order to achieve an optimal value for $U_{i}(\Psi)$, the players will negotiate and change their interdependent strategies in $\Psi$. Then, two important issues will arise: (i) whether they will ever reach a consensus, or a steady state, and (ii) if the steady state exists, whether the steady state of the game is also the precise localization of the whole network.

Definition 1 (Nash equilibrium, NE). A strategy $s^{*} \in \Psi$ is a NE if it satisfies

$$
U_{i}\left(s^{*}\right) \leq U_{i}\left(s_{i}^{\prime}, s_{-i}\right), \quad \forall s_{i}^{\prime} \in S, \forall i \in A .
$$

According to this definition, no player can benefit by deviating from its strategy if other players do not change theirs. In other words, this result guarantees an agreement for negotiations among all the players. However, no optimal outcome or fairness is intrinsically guaranteed. Nevertheless, we show that the sensor location game falls into the category of potential games, where the existence of Nash equilibrium can be established.

Definition 2 (potential game). A game is defined as a potential game in which a potential function $P$ exists, such that

$$
P\left(s_{i}, s_{-i}\right)-P\left(s_{i}^{\prime}, s_{-i}\right)=U_{i}\left(s_{i}, s_{-i}\right)-U_{i}\left(s_{i}^{\prime}, s_{-i}\right), \quad \forall i,
$$

where $s_{i}$ and $s_{i}^{\prime}$ are two arbitrary strategies.

Potential game is a specific type of games. And if a game is a potential one, there exists at least one pure strategy NE for such a game. Moreover, all NEs are either local or global optimal solutions of the potential function $P$.

Theorem 3. Nonanchor node localization game is a potential game, and has a Nash equilibrium, which minimizes, either locally or globally, the following function:

$$
P\left(s_{i}, s_{-i}\right)=\frac{1}{2} * R(\Psi) .
$$

Proof. Without loss of generality, let us assume sensor node $i$ is updating its location selection unilaterally, given the location selections of other sensors, that is, $s_{-i}$. For two feasible location selection strategies $s_{i}$ and $s_{i}^{\prime}$, the performance difference for sensor node $i$ is calculated as

$$
U_{i}\left(s_{i}, s_{-i}\right)-U_{i}\left(s_{i}^{\prime}, s_{-i}\right)=\sum_{j \in N_{i}} \delta\left(\widehat{p}_{i}, \widehat{p}_{j}\right)-\sum_{j \in N_{i}} \delta\left(\widehat{p}_{i}^{\prime}, \widehat{p}_{j}\right) .
$$


We next rewrite the function $P$ and obtain

$$
\begin{aligned}
P\left(s_{i}, s_{-i}\right)= & \frac{1}{2} * \sum_{i=1}^{N_{n}} \sum_{j \in N_{i}} \delta\left(\widehat{p}_{i}, \widehat{p}_{j}\right) \\
= & \frac{1}{2} *\left(\sum_{j \in N_{i}} \delta\left(\widehat{p}_{i}, \widehat{p}_{j}\right)+\sum_{k \neq i, k=1}^{N_{n}} \sum_{j \in N_{k}} \delta\left(\widehat{p}_{k}, \widehat{p}_{j}\right)\right) \\
= & \frac{1}{2} *\left(\sum_{j \in N_{i}} \delta\left(\widehat{p}_{i}, \widehat{p}_{j}\right)\right. \\
& +\sum_{k \neq i, k=1}^{N_{n}}\left(\sum_{j \neq i, j \in N_{k}} \delta\left(\widehat{p}_{k}, \widehat{p}_{j}\right)\right. \\
= & \frac{1}{2} *\left(\sum_{j \in N_{i}} \delta\left(\widehat{p}_{i}, \widehat{p}_{j}\right)+\sum_{k \neq i, k=1}^{N_{n}} \sum_{j \neq i, j \in N_{k}} \delta\left(\widehat{p}_{k}, \widehat{p}_{j}\right)\right. \\
& \left.+\sum_{k \neq i, k=1}^{N_{n}} \delta\left(\widehat{p}_{k}, \widehat{p}_{i}\right)\right) .
\end{aligned}
$$

According to (4), if nodes $k$ and $i$ are not neighbors, $\delta\left(\widehat{p}_{k}, \widehat{p}_{i}\right)=0$; then we have

$$
\begin{aligned}
\sum_{k \neq i, k=1}^{N_{n}} \delta\left(\widehat{p}_{k}, \widehat{p}_{i}\right)= & \sum_{k \in N_{i}} \delta\left(\widehat{p}_{k}, \widehat{p}_{i}\right) \\
& +\sum_{k \neq i, k \notin N_{i}, k=1}^{N_{n}} \delta\left(\widehat{p}_{k}, \widehat{p}_{i}\right)=\sum_{k \in N_{i}} \delta\left(\widehat{p}_{k}, \widehat{p}_{i}\right) .
\end{aligned}
$$

And (13) can be rewritten as

$$
\begin{aligned}
P\left(s_{i}, s_{-i}\right)=\frac{1}{2} *\left(\sum_{j \in N_{i}} \delta\left(\widehat{p}_{i}, \widehat{p}_{j}\right)\right. & \\
& +\sum_{k \neq i, k=1}^{N_{n}} \sum_{j \neq i, j \in N_{k}} \delta\left(\widehat{p}_{k}, \widehat{p}_{j}\right) \\
& \left.+\sum_{k \in N_{i}} \delta\left(\widehat{p}_{k}, \widehat{p}_{i}\right)\right) \\
=\frac{1}{2} *\left(2 * \sum_{j \in N_{i}} \delta\left(\widehat{p}_{i}, \widehat{p}_{j}\right)\right. & \\
& \left.+\sum_{k \neq i, k=1}^{N_{n}} \sum_{j \neq i, j \in N_{k}} \delta\left(\widehat{p}_{k}, \widehat{p}_{j}\right)\right)
\end{aligned}
$$

$$
\begin{aligned}
= & \sum_{j \in N_{i}} \delta\left(\widehat{p}_{i}, \widehat{p}_{j}\right) \\
& +\frac{1}{2} * \sum_{k \neq i, k=1}^{N_{n}} \sum_{j \neq i, j \in N_{k}} \delta\left(\widehat{p}_{k}, \widehat{p}_{j}\right) .
\end{aligned}
$$

Thus, the difference of function $P$ with two strategies $s_{i}$ and $s_{i}^{\prime}$ can be calculated as

$$
\begin{aligned}
P\left(s_{i}, s_{-i}\right)-P\left(s_{i}^{\prime}, s_{-i}\right) & \\
= & \sum_{j \in N_{i}} \delta\left(\widehat{p}_{i}, \widehat{p}_{j}\right)+\frac{1}{2} * \sum_{k \neq i, k=1}^{N_{n}} \sum_{j \neq i, j \in N_{k}} \delta\left(\widehat{p}_{k}, \widehat{p}_{j}\right) \\
& -\left(\sum_{j \in N_{i}} \delta\left(\widehat{p}_{i}^{\prime}, \widehat{p}_{j}\right)+\frac{1}{2} * \sum_{k \neq i, k=1}^{N_{n}} \sum_{j \neq i, j \in N_{k}} \delta\left(\widehat{p}_{k}, \widehat{p}_{j}\right)\right) \\
= & U_{i}\left(s_{i}, s_{-i}\right)-U_{i}\left(s_{i}^{\prime}, s_{-i}\right) .
\end{aligned}
$$

Note that we utilize the property that when a single sensor node $i$ switches its location strategy from $s_{i}$ to $s_{i}^{\prime}$, the costs of other sensors that are not neighbors of $i$ are unchanged. We stress that the above equation is valid for any $i, s_{i}$ to $s_{-i}$. Therefore, the location selection game is an exact potential game with a potential function given by (11). It is worth noting that every Nash equilibrium in the location selection game, where no sensor node can improve its own performance by deviating unilaterally, corresponds to a local or global localization solution of the whole network. The existence of Nash equilibrium follows the results of [25].

By making use of $\mathrm{NE}$ and potential game, our localization approach can converge to an agreement among all players and this point is an efficient solution of the whole network. In the literature, there are two famous learning schemes that are used to converge to NE, namely, the best and better response techniques, expressed as follows:

$$
\begin{aligned}
& s_{i}^{t+1}=\underset{s \in S_{i}}{\arg \min } U_{i}(\Psi) \\
&=\underset{s \in S_{i}}{\arg \min }\left(\sum_{j \in N_{i}, j \in N_{n}}\left(d_{i j}-\left\|s-s_{j}^{t}\right\|\right)^{2}\right. \\
&\left.+\sum_{j \in N_{i}, j \notin N_{n}}\left(d_{i j}-\left\|s-p_{j}\right\|\right)^{2}\right), \\
& s_{i}^{t+1}= \begin{cases}s_{i}^{\text {rand }}, & \text { if } U_{i}\left(s_{i}^{\text {rand }}, s_{-i}\right)<U_{i}\left(s_{i}^{t}, s_{-i}\right), \\
s_{i}^{t}, & \text { otherwise. }\end{cases}
\end{aligned}
$$

In the former scheme, for each player, during its turn to play, it searches its entire strategy space and selects the one that yields the best outcome considering all its neighbors' (including both neighbor anchor node and neighbor nonanchor node) strategies, as shown in (17). The primary drawback 
of the best response is the computational complexity for resource constrained sensor nodes, which grows linearly with the cardinality of the strategy space. The latter scheme is an improvement of the former one, where at each step, the player updates its strategy as long as the randomly selected strategy $s_{i}^{\text {rand }}$ yields a better utility $U_{i}\left(s_{i}^{\text {rand }}, s_{-i}\right)$ than the previous one $U_{i}\left(s_{i}^{t}, s_{-i}\right)$, as shown in (18). The dramatically reduced computation is the trade off with the convergence speed. Both the best response and the better response are guaranteed to converge to a NE in potential games [25]. However, the equilibrium may occur at the local optimum of the utility function, instead of the global optimum if there are multiple NEs. In this case, the system performance will be trapped in a suboptimal state and, since this is one instance of NE, no node will be able to increase its utility function by changing its strategy. Therefore, some response techniques should be designed to avoid being trapped in a local optimal state.

In our paper, the smoothed better response learning scheme [26] is applied, which can converge to the optimal NE with high probability. In this scheme, for each player $i$, during its turn to play, it selects a random strategy $s_{i}^{\text {rand }}$ and keeps it with the probability of $p\left(s_{i}^{\text {rand }}, s_{i}^{t}\right)$, which can be expressed as

$$
p\left(s_{i}^{\text {rand }}, s_{i}^{t}\right)=\frac{1}{1+\mathrm{e}^{\left(U_{i}\left(s_{i}^{\text {rand }}, s_{-i}\right)-U_{i}\left(s_{i}^{t}, s_{-i}\right)\right) / \gamma}},
$$

where $\gamma$ is the smoothing factor. Equation (19) is a function of the difference of the utility functions; if $U_{i}\left(s_{i}^{\text {rand }}, s_{-i}\right)<$ $U_{i}\left(s_{i}^{t}, s_{-i}\right)$, player $i$ will change its strategies with high probability; otherwise, it will keep them with high probability. If only a small difference occurs, the player will select one of the strategies almost randomly. In this case, even though the players will be able to select a "worse" strategy or not to select a marginally "better" strategy, this uncertainty of the probability allows the players to move from a local optimum state and to start negotiating towards a new NE.

In addition, smoothing factor $\gamma$ is responsible for controlling the trade-off between the algorithmic performance and convergence speed. The larger the smoothing factor is, the more extensive strategy search and slower convergence speed will be obtained. On the other hand, smaller $\gamma$ represents more limited search and faster a convergence speed. In our simulations, we borrow the concept of temperature in simulated annealing [18], and $\gamma$ is set to $10 / t^{2}$, where $t$ denotes the negotiation iterations. It is advisable that $\gamma$ keeps deceasing as the negotiation iterates.

Due to the uneven sensor distribution, some nonanchor nodes with more neighbor anchors and larger neighbor connectivity may tend to converge faster than others. With the purpose of saving energy during negotiation, those nodes that are already accurately localized should be detected and deleted from the game as early as possible. Moreover, any time when a nonanchor node is considered to be localized, it should elevate as an anchor node and help to decide the strategy space for all its neighbor nonanchor nodes. In our paper, we further propose the following strategy space determination techniques for each player before game negotiation.

(i) If the player has three or more neighbor anchors, the estimate on the position of this sensor can be calculated by one of the existing algorithms for range based positioning, such as the one proposed in [27], and the player is elevated as an anchor node.

(ii) If the sensor has two neighbor anchors, its strategy space is set as the junctions of its neighbor anchors' communication circles.

(iii) If the sensor has one neighbor anchor, its strategy space is set as the communication circle of its neighbor anchor.

(iv) If the sensor has no neighbor anchor, its strategy space is set as the full target area.

In addition, after game negotiation, if the value of the utility function of one player is smaller than the prior given localization threshold, it may be precisely localized or localized with flip-ambiguity [15]. In order to avoid the flip-ambiguity during localization, only the value of the utility function achieved by the player is satisfactory in consecutive $k$ times; the player is considered to be accurately localized. And then this player is elevated as an anchor node. Combined with the parameters and techniques associated with our localization game discussed above, the steps of the proposed localization algorithm are summarized in Algorithm ??. For each player, its game framework involves a strategy space generation step, a negotiation step, and an anchor node elevation step, where only local communications are necessary in all steps.

In our algorithm, $T$ is the maximum number of iterations for localization game and $w_{i}$ records the number of consecutive game iterations that the $i$ th node's utility function is no larger than $\Theta_{h}$. Once $w_{i} \geq k$, node $i$ is considered to be accurately localized and is elevated as an anchor node. It is obvious that, for these nodes elevated as anchor nodes, their game iterations are smaller than $T$, and their complexity is smaller than $O\left(n_{c} * T\right)$, where $n_{c}$ is the average size of their neighbor sensor set. Therefore the time complexity of the whole network is $O\left(N_{n} * n_{c} * T\right)$.

\section{Performance Evaluation}

To evaluate the performance of the proposed algorithm, we introduce the mean localization error (MLE), which can be calculated by

$$
\text { MLE }=\frac{1}{N_{n}} \sqrt{\sum_{i=1}^{N_{n}}\left\|\hat{p}_{i}-p_{i}\right\|^{2}} .
$$

4.1. Simulation in Regular Network. At first, 200 nonanchor nodes and 10 anchor nodes are randomly located in a $100 \times$ 100 area network. The communication range is set to 15 , the variance of measurement error $e_{i j}$ is given by $\lambda^{2} r_{i j}^{2}$, at first, $\lambda$ is first set to 0 , which means that the system is in an ideal situation, and there is no measurement error in distance calculation.

Figure 1(a) shows the initial distribution and the nonanchor nodes connecting with anchor nodes, where the anchors and the true locations of nonanchor nodes are shown by red asterisks and green circles. Figure 1(b) shows the final 
(1) set $t=0, s_{i}=\{\emptyset\}, w_{i}=0(\forall i \in A)$

(2) while $t<T$

(3) for each non-anchor node $i \in A$

(4) //solution space generation

(5) get posOfNeighborsandnumOfNeighborAnchor of $i$

(6) if numOfNeighborAnchor $<1$

(7) set its straSpace as the target area

(8) else if numOfNeighborAnchor equals to 1

(9) set its straSpace as the communication circle of its anchor

(10) else if numOfNeighborAnchor equals to 2

(11) set its straSpace as the junctions of its neighbor anchors' communication circles

(12) else

(13) estimate the position of the player using existing localization algorithms

(14) $\quad A=A-\{i\}$;

(15) continue;

(16) end if

(17) // negotiation

(18) $s_{i}^{\text {rand }} \leftarrow$ random strategy form its strategy space;

(19) if $p\left(s_{i}^{\text {rand }}, s_{i}^{t}\right) \geq$ random number from 0 to 1

(20) $s_{i}^{t+1} \leftarrow s_{i}^{\text {rand }}$

(21) else

(22) $s_{i}^{t+1} \leftarrow s_{i}^{t}$

(23) end if

(24) // anchor node elevation

(25) if $U_{i}\left(s_{i}^{t+1}, s_{-i}\right) \leq \Theta_{h}$

(26) $\quad w_{i}=w_{i}+1$;

(27) if $w_{i} \geq k$

(28) Elevate node $i$ as an anchor node

(29) $A=A-\{i\}$;

(30) else

(31) $w_{i}=0$

(32) $\quad t=t+1$

(33) end if

(34) end for

(35) end while

Algorithm 1: Distributed localization algorithm based on potential game.

location results after 500th iterations, where the anchors and the true locations of nonanchor nodes are also shown by red asterisks and green circles, the estimated locations are shown by the plus, and the dashed lines quantize localization error. From the simulation results in Figure 1, we can make the following observations. (1) As can be observed in Figure 1(a), no more than 20 nodes have two anchor neighbors, and the other anchor nodes have less than one anchor neighbor. (2) Almost all the nonanchor nodes are accurately localized in our algorithm; only a few nodes are deviated from the true locations, as can be observed in Figure 1(b). This is mainly because of the nonuniqueness of these nodes' location in the network, where the flip-ambiguity problem is happening during localization game.

In Figure 2, we illustrate the negotiation process reaching the NE with different responses. In the best response, the player selects the strategy with the best utility function, which has fast convergence speed as expected, but it may generate suboptimal results when nodes find themselves trapped in a local optimum NE. In contrast, better response can obtain better results than the best response, but it needs many more iterations to converge. As the uncertainty is used in strategy selection, the SBR scheme has the best performance. And we also can find that, by adding the accelerating techniques in the localization game, both strategies can converge to the NE rapidly.

Since our approach is a range based technique, in a practical application, the measurement error has great influence on the localization accuracy. We further set communication radius as 15,20 , and 25 and compare the localization performance with different measurement error. Considering that the road sensor network is usually deployed in a typical outdoor environment, the measurement error is smaller than 0.1 in most cases [15]. Therefore, in our simulation, the range of $\lambda$ is set from 0 to 0.2 , and simulations with $\lambda>0.2$ are not executed in our paper.

Figure 3 shows the MLE comparison with different communication radii and different $\lambda$. From Figure 3, we can draw the conclusion that (1) localization accuracy will be improved when the communication radius increases; this is 


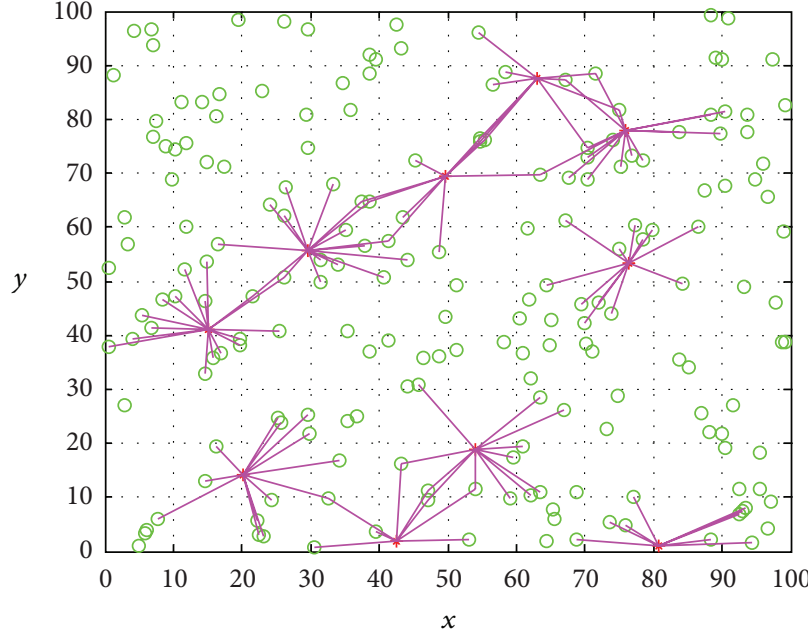

(a) Initial distribution

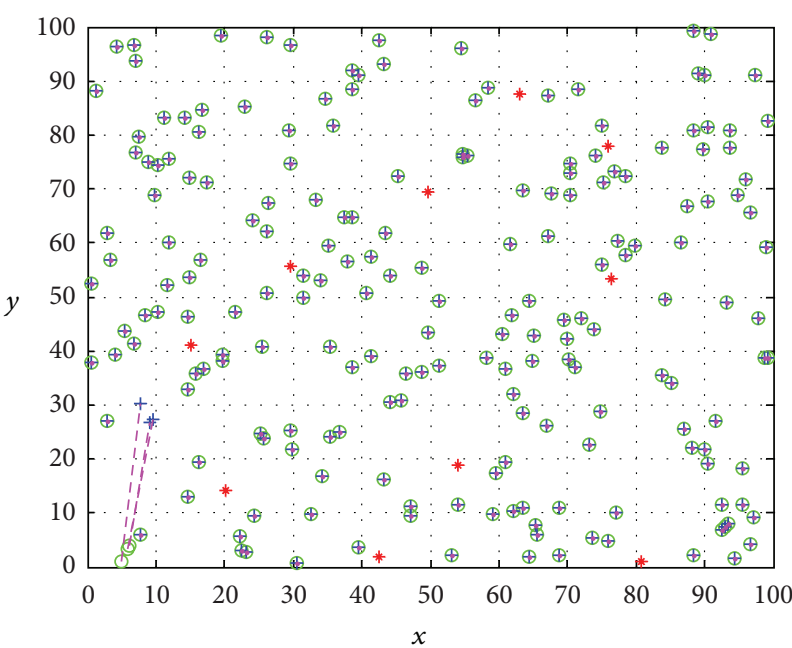

(b) Final localization results

FIgURE 1: The initial distribution and final localization results.

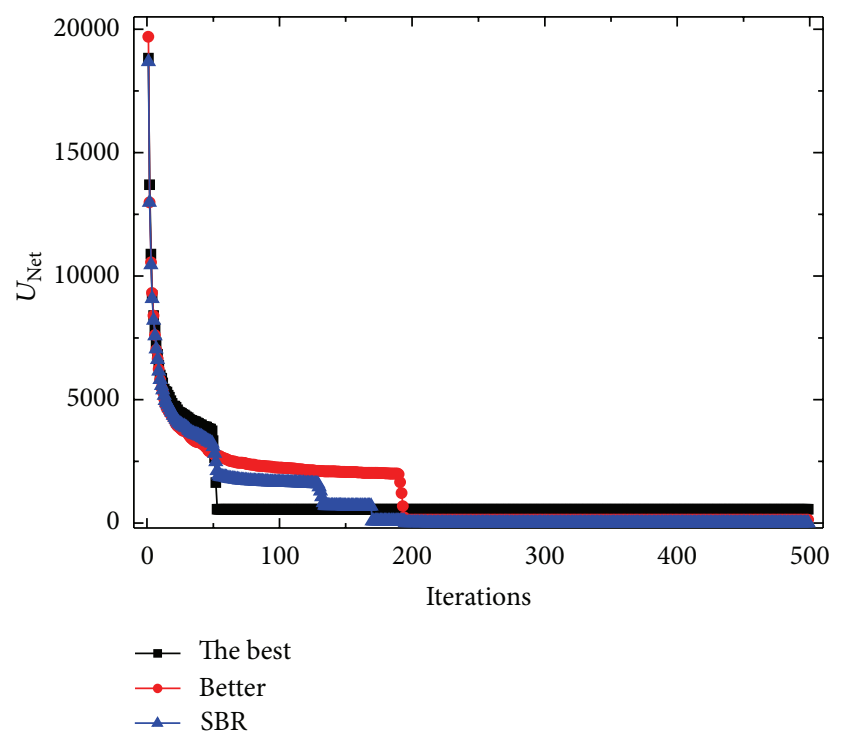

FIGURE 2: $U_{\text {Net }}$ in different numbers of iterations.

mainly because the larger the communication radius is, the more node connectivity can be obtained, thus improving the localization performance; (2) localization error increases with the measurement error increases; (3) when the communication radius increases to 25 and 30, our work can work well for typical outdoor environment $(\lambda>0.1)$; (4) even when the measurement error is very large, our algorithm still works better, which demonstrates the effectiveness of the algorithm.

4.2. Simulation in Irregular Network. In a practical road sensor network deployment, it is most likely that the shape of the roadway is irregular due to the presence of physical obstacles. In Figure 4, we give an example of such a network, in particular, a C-shaped network, with 200 nonanchor nodes and 10 anchors.

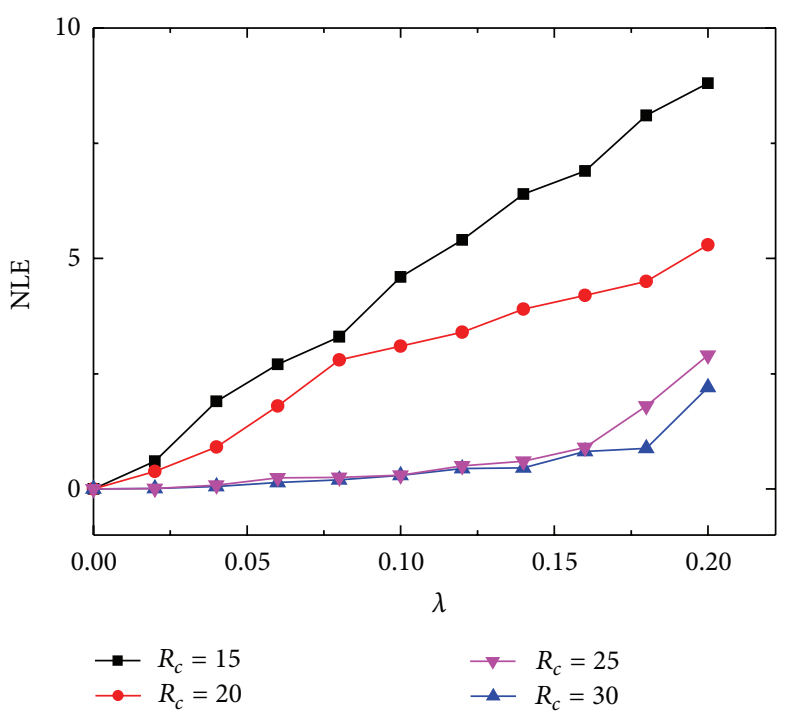

FIgURE 3: MLE comparison with different communication radii and different $\lambda$ in regular networks.

Figure 5 shows the MLE comparison with different communication radii and different $\lambda$. From Figure 5, we can draw the conclusion that (1) localization error is more serious in irregular networks shapes than that in regular networks; (2) when the communication range is larger than 25, for about $60 \%$ of the scenarios, the localization error is less than 2, which demonstrates the robustness against distance estimation noise and irregular network shape.

4.3. Comparison with MDS-MAP and PAES. We further compare the performance obtained by our algorithm with those obtained by the PAES algorithm and MDS-MAP algorithm with the same network topologies, where the target area is set to $100 * 100, R_{c}$ is set to 25 , and $\lambda$ is set to 0.1 . 


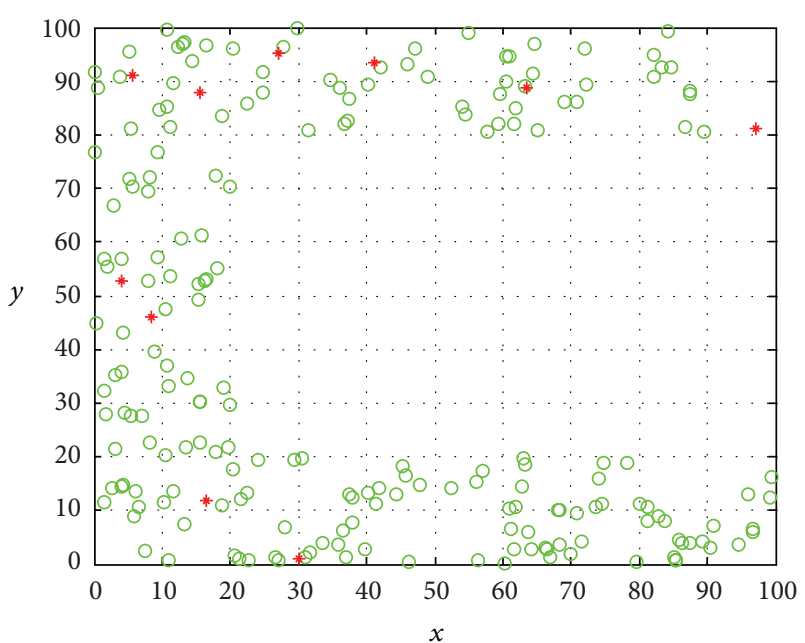

FIGURE 4: Irregular sensor distribution.

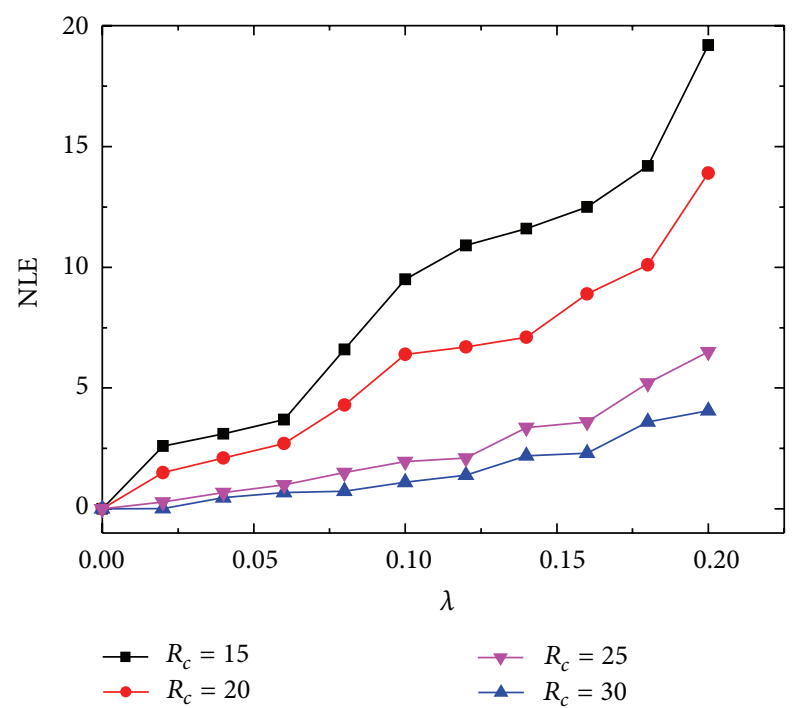

FIGURE 5: MLE comparison with different communication radii and different $\lambda$ in irregular networks.

In each topology, the ratio of anchor nodes and nonanchor nodes is 0.1. Since our algorithm and PAES are running with the number of iterations, the maximum number iterations of these two algorithms is 1000 , and any time the total utility function decreases to 0.5 , the whole network is considered accurately localized and the total running time is calculated.

Table 1 shows the average localization error and computation time of the 6 network topologies with different numbers of sensor nodes. From Table 1, we can get some conclusions as follows: (1) our algorithm has a significantly shorter running time and localization error than MDS-MAP and PAES in most cases $\left(N_{n} \geq 100\right)$, which is mainly because in our approach, its complexity scales as $O\left(N_{n} * n_{c} * T\right)$; (2) the time complexities of PAES and MDA-MAP are $O\left(N_{n} * N_{n} *\right.$ $T)$ and $O\left(N_{n} * n_{c}^{3}\right)$, as PAES takes a number of iterations much larger than $n$ to converge; that is, $T \gg n$; PAES
TABLE 1: Comparison of localization error and computation time.

\begin{tabular}{lccc}
\hline$N_{n}$ & Algorithm & NLE (m) & Computation time (s) \\
\hline \multirow{3}{*}{50} & Game & 6.18 & 0.07 \\
& MDS-MAP & 5.28 & 0.03 \\
& PAES & 7.61 & 0.59 \\
\hline \multirow{3}{*}{100} & Game & 1.37 & 0.11 \\
& MDS-MAP & 6.25 & 0.18 \\
& PAES & 3.32 & 1.23 \\
\hline \multirow{3}{*}{150} & Game & 0.32 & 0.17 \\
& MDS-MAP & 1.09 & 0.35 \\
& PAES & 1.05 & 2.1 \\
\multirow{4}{*}{200} & Game & 0.29 & 0.27 \\
& MDS-MAP & 3.57 & 0.62 \\
& PAES & 0.82 & 3.66 \\
\multirow{3}{*}{250} & Game & 0.32 & 0.33 \\
& MDS-MAP & 3.4 & 1.11 \\
& PAES & 0.68 & 4.7 \\
\multirow{3}{*}{300} & Game & 0.22 & 0.35 \\
& MDS-MAP & 3.47 & 1.7 \\
& PAES & 0.34 & 5.21 \\
\hline \multirow{3}{*}{0} & & &
\end{tabular}

takes the longest computation time to converge; (3) when the number of deployed nodes is very small $\left(N_{n} \leq 50\right)$, our algorithm has a slightly longer running time and localization error than MDS-MAP, which is mainly because when the number of sensors is very small, each nonanchor node has fewer neighbor sensors, and thus more nodes tend to be flipped during localization and more running time is used for convergence. Combined with our previous results on the localization error for different network shapes, our proposed localization algorithm is a very efficient and distributed method with excellent localization accuracy.

\section{Conclusion}

In this paper, we have proposed a potential game for distributed localization for road sensor networks using only local information. Our main contributions are as follows. First, we have transformed the optimal localization problem to a distributed potential game. Second, we have developed a negotiation-based localization algorithm using the properties of potential game. Furthermore, we have derived new methods to accelerate the localization algorithm in converging to optimal solutions. Extensive simulations demonstrate that our algorithm can quickly converge to the optimal solution and perform well even with a small number of anchor nodes and achieve better localization accuracy and efficiency than the existing algorithms.

\section{Acknowledgments}

This work is supported by the National Science Foundation for Distinguished Young Scholars of China under Grant no. 61225012 and no. 71325002; the National Natural Science Foundation of China under Grant no. 61173153 and no. 
60903159; the Fundamental Research Funds for the Central Universities under Grant nos. N110404014, N110318001, N110204003, and N120104001; the Specialized Research Fund of the Doctoral Program of Higher Education for the Priority Development Areas under Grant no. 20120042130003; the Specialized Research Fund for the Doctoral Program of Higher Education under Grant no. 20110042110024; the China Postdoctoral Science Foundation funded project under Grant no. 20110491508 and no. 2012T50248.

\section{References}

[1] I. F. Akyildiz, W. Su, Y. Sankarasubramaniam, and E. Cayirci, "Wireless sensor networks: a survey," Computer Networks, vol. 38, no. 4, pp. 393-422, 2002.

[2] M. Shuai, K. Xie, M. Xiujun, and G. Song, "An on-road wireless sensor network approach for urban traffic state monitoring," in Proceedings of the 11th International IEEE Conference on Intelligent Transportation Systems (ITSC '08), pp. 1195-1200, Beijing , China, December 2008.

[3] R. J. Weiland and L. B. Purser, "Intelligent transportation systems," in Transportation in the New Millennium, 2000, (030001).

[4] "Driving Today: Cars Talking to Cars," http://www.drivingtoday.com/partner/content/StudioOne/archive/feature/cars_ talking_to_cars/index.html.

[5] Toyota Motor Corporation (TMC), "TMC Develops Onboard DSRC Unit to Improve Traffic Safety," http://www2.toyota.co .jp/en/news/09/09/0903.html.

[6] European Telecommunications Standards Institute (ETSI), "Intelligent Transportation Systems," http://www.etsi.org/ WebSite/technologies/IntelligentTransportSystems.aspx.

[7] G. Mao, B. Fidan, and B. D. O. Anderson, "Wireless sensor network localization techniques," Computer Networks, vol. 51, no. 10, pp. 2529-2553, 2007.

[8] J. Jia, X. Wu, J. Chen, and X. Wang, "Exploiting sensor redistribution for eliminating the energy hole problem in mobile sensor networks," EURASIP Journal on Wireless Communications and Networking, vol. 2012, article 68, 2012.

[9] J. Jia, J. Chen, X. Wang, and L. Zhao, "Energy-balanced density control to avoid energy hole for wireless sensor networks," International Journal of Distributed Sensor Networks, vol. 2012, Article ID 812013, 10 pages, 2012.

[10] A. Vempaty, O. Ozdemir, K. Agrawal, H. Chen, and P. K. Varshney, "Localization in wireless sensor networks: byzantines and mitigation techniques," IEEE Journal and Magazines, vol. 61, no. 6, pp. 1495-1508, 2013.

[11] X. Li, "RSS-based location estimation with unknown pathloss model," IEEE Transactions on Wireless Communications, vol. 5, no. 12, pp. 3626-3633, 2006.

[12] A. Savvides, C.-C. Han, and M. B. Strivastava, "Dynamic fine-grained localization in ad-hoc networks of sensors," in Proceedings of the 7th Annual International Conference on Mobile Computing and Networking, pp. 166-179, July 2001.

[13] X. Li and K. Pahlavan, "Super-resolution TOA estimation with diversity for indoor geolocation," IEEE Transactions on Wireless Communications, vol. 3, no. 1, pp. 224-234, 2004.

[14] L. Doherty, K. S. J. Pister, and L. El Ghaoui, "Convex position estimation in wireless sensor networks," in Proceedings of the 20th Annual Joint Conference of the IEEE Computer and Communications Societies, pp. 1655-1663, Anchorage, Alaska, USA, April 2001.
[15] M. Vecchioa, R. L. López, and F. Marcellonib, "A two-objective evolutionary approach based on topological constraints for node localization in wireless sensor networks," Applied Soft Computing, vol. 15, no. 7, pp. 1891-1901, 2012.

[16] Y. Shang, W. Ruml, Y. Zhang, and M. Fromherz, "Localization from connectivity in sensor networks," IEEE Transactions on Parallel and Distributed Systems, vol. 15, no. 11, pp. 961-974, 2004.

[17] P. Biswas and Y. Ye, "Semidefinite programming for ad hoc wireless sensor network localization," in Proceedings of the $3 \mathrm{rd}$ International Symposium on Information Processing in Sensor Networks (IPSN '04), pp. 46-54, April 2004.

[18] A. A. Kannan, G. Mao, and B. Vucetic, "Simulated annealing based wireless sensor network localization with flip ambiguity mitigation," in Proceedings of the IEEE 63rd Vehicular Technology Conference (VTC '06), pp. 1022-1026, Melbourne, Australia, July 2006.

[19] P. Biswas, T.-C. Liang, K.-C. Toh, Y. Ye, and T.-C. Wang, "Semidefinite programming approaches for sensor network localization with noisy distance measurements," IEEE Transactions on Automation Science and Engineering, vol. 3, no. 4, pp. 360-371, 2006.

[20] P. Biswas, T.-C. Lian, T.-C. Wang, and Y. Ye, "Semidefinite programming based algorithms for sensor network localization," ACM Transactions on Sensor Networks, vol. 2, no. 2, pp. 188220, 2006.

[21] J. A. Costa, N. Patwari, and A. O. Hero III, "Distributed weighted-multidimensional scaling for node localization in sensor networks," ACM Transactions on Sensor Networks, vol. 2, no. 1, pp. 39-64, 2006.

[22] N. Gatti, M. Monga, and S. Sicari, "A localization game in wireless sensor networks," in Proceedings of the International Conference on Decision and Game Theory for Security, pp. 168179, 2010.

[23] B. Bejar, P. Belanovic, and S. Zazo, "Cooperative localisation in wireless sensor networks using coalitional game theory," in Proceedings of the 18th European Signal Processing Conference, pp. 1459-1463, 2010.

[24] S. M. Kay, Fundamentals of Statistical Signal Processing: Estimation Theory, Prentice-Hall, 1993.

[25] D. Monderer and L. S. Shapley, "Potential games", Games and Economic Behavior, vol. 14, no. 1, pp. 124-143, 1996.

[26] G. Arslan, M. F. Demirkol, and Y. Song, "Equilibrium efficiency improvement in MIMO interference systems: a decentralized stream control approach," IEEE Transactions on Wireless Communications, vol. 6, no. 8, pp. 2984-2993, 2007.

[27] S. Zhu and Z. Ding, "A simple approach of range-based positioning with low computational complexity," IEEE Transactions on Wireless Communications, vol. 8, no. 12, pp. 5832-5836, 2009. 


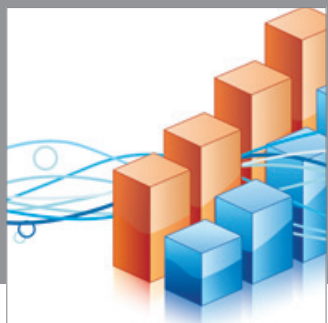

Advances in

Operations Research

mansans

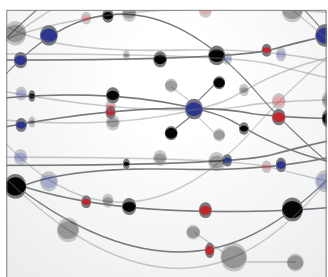

The Scientific World Journal
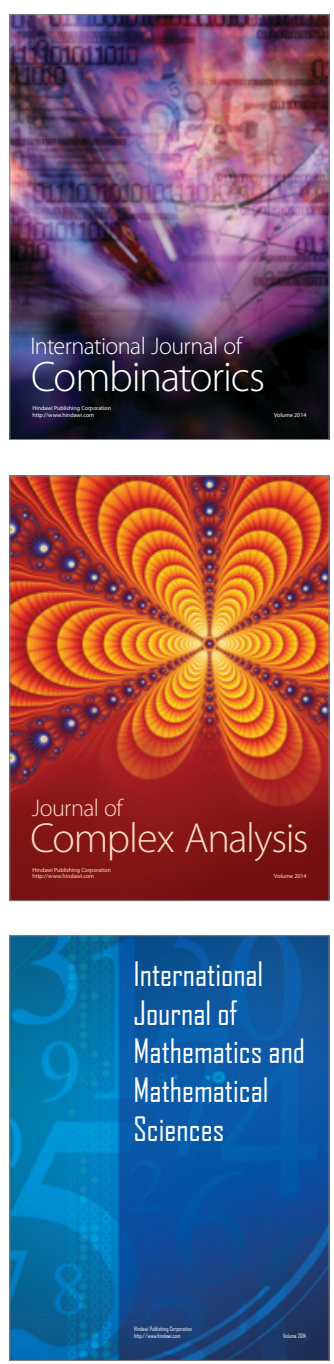
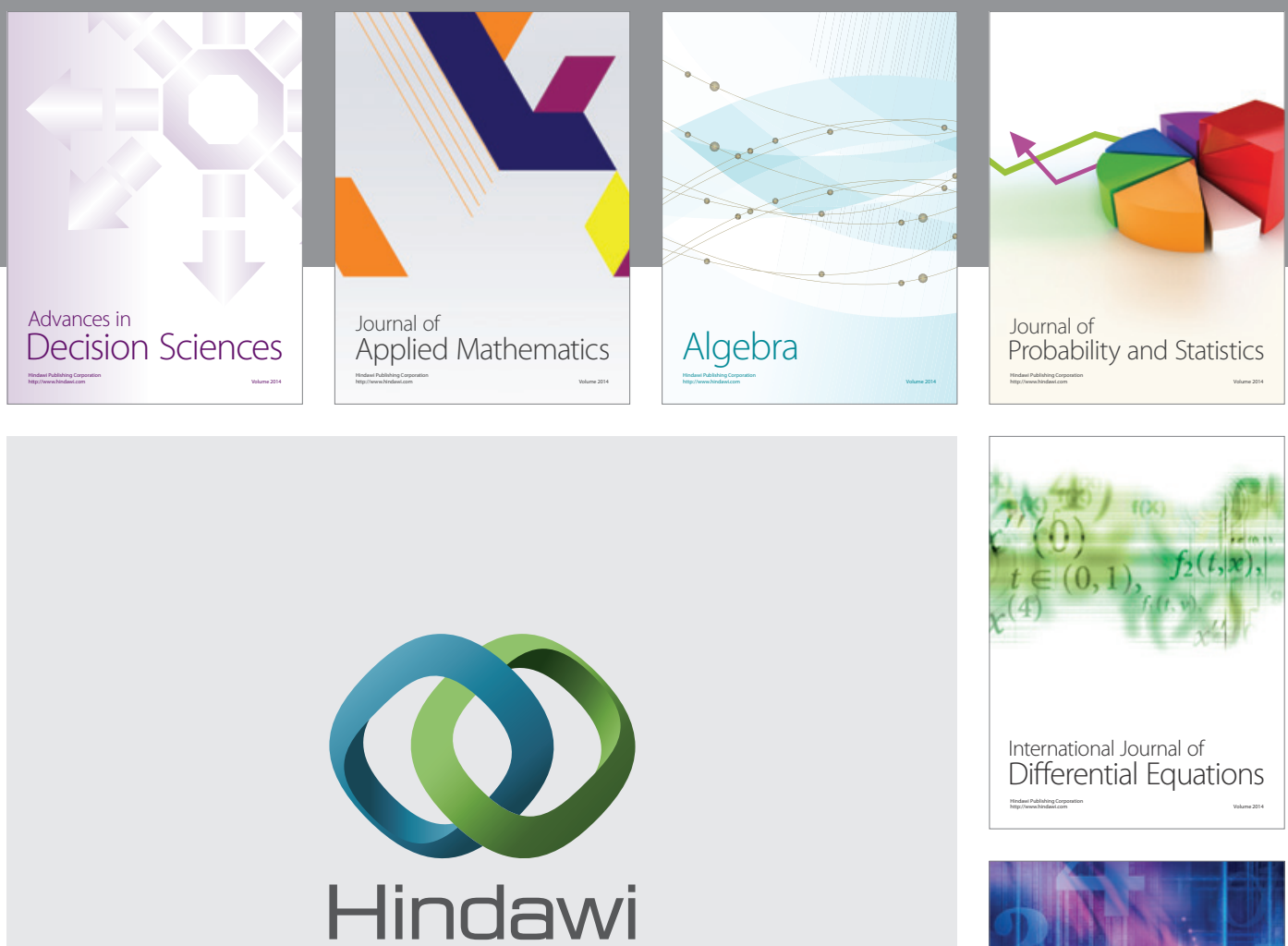

Submit your manuscripts at http://www.hindawi.com
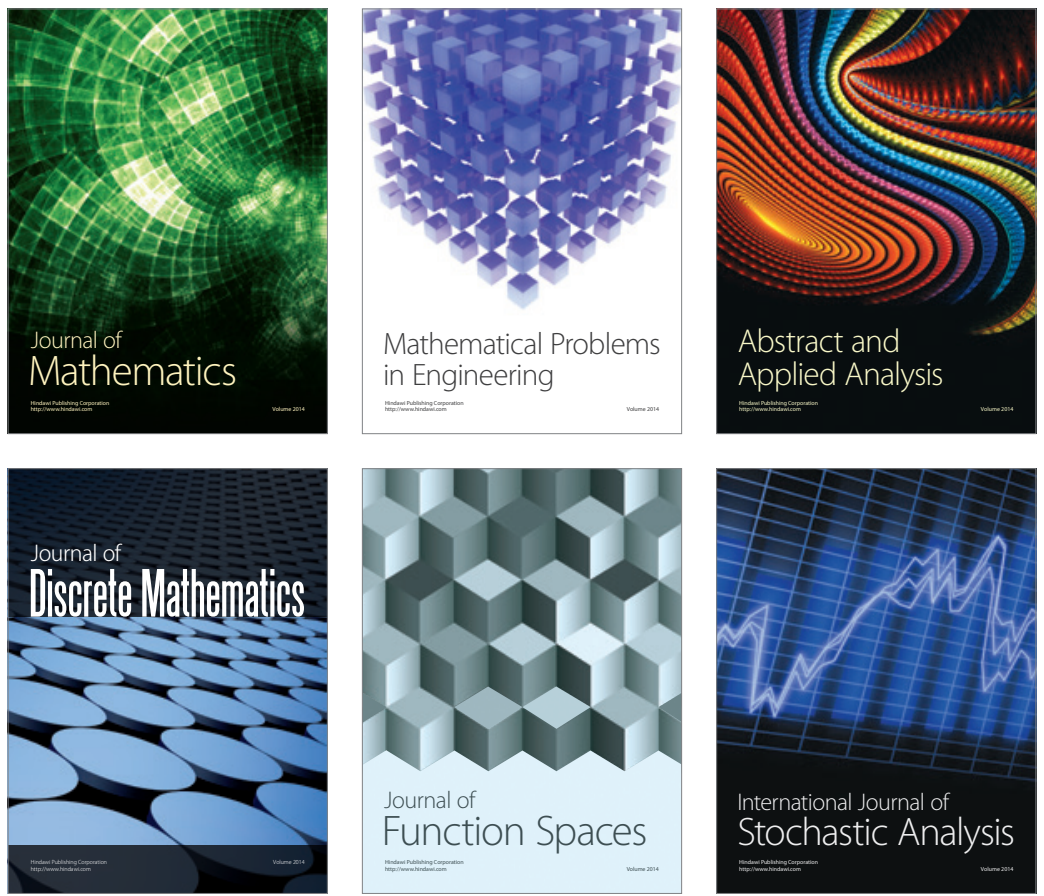

Journal of

Function Spaces

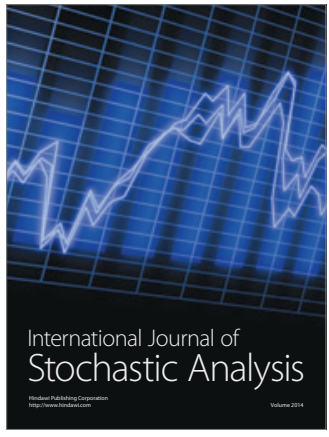

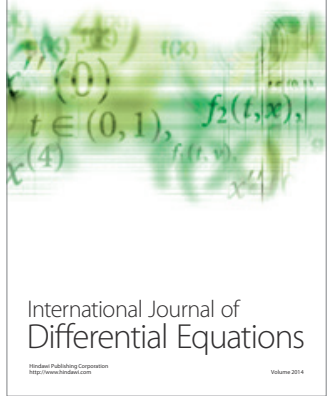
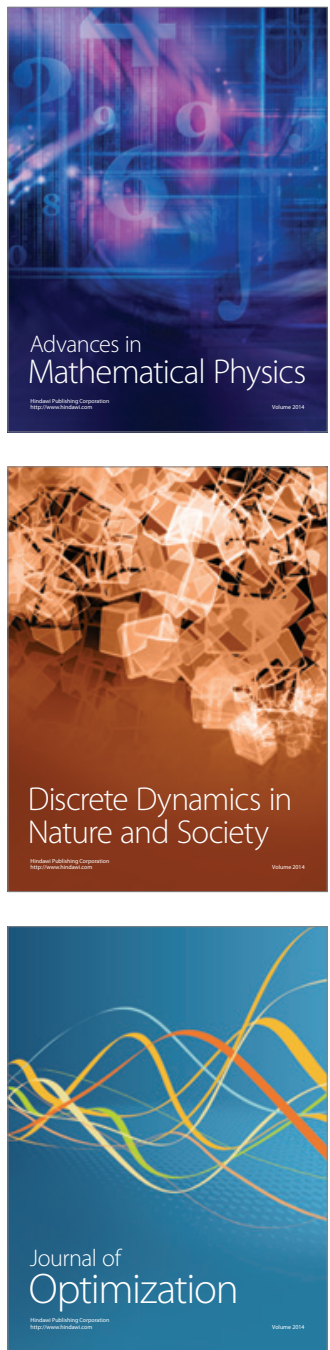